\title{
A Multi-Agent-based Communication Prototype for Cross-Company Capacity Exchange in Manufacturing Networks
}

\author{
Manufacturing companies face tough challenges in leveling their capacity load \\ due to unsteady market conditions, like fluctuating demand. This induces \\ frequent re-scheduling in order to harmonize the capacity utilization of machine \\ tools to meet customer requirements in terms of delivery time, costs, and product \\ quality. In most cases an internal adjustment is not possible, so that a \\ subcontracting is unavoidable. This requires significant efforts and can be very \\ time and cost intensive. So, there is a need for a system supporting the cross- \\ company capacity exchange which is yet not addressed in both literature and \\ industry. This paper deals with the development of such a capacity exchange \\ system and presents first result of its application.
}

Keywords: capacity; exchange; multi-agent system; networks

\section{Problem Statement}

Manufacturing companies operate more and more in an increasingly complex market environment with fluctuating demand in terms of quantity, type, and delivery time, as well as an increasing division of labor with a specialization in core competencies. The short-term demand changes and new demand requests can result in excessing or dropping below physical capacity limits of resources as well as rejecting additional requests due to lack of competencies.

In several cases, an internal temporal adaptation of this additional capacity requirement is hardly possible in compliance with the delivery times requested by customers. So, companies are encouraged to acquire additional competencies and capacities. One possibility for companies to meet these requirements and to compensate machines capacities in short-term over or under load situations consists of short-term capacity exchange in terms of outtasking of corresponding processes if internal capacity adaptation measures are not sufficient. 
The main problem, however, is the time required for the initiation of such cooperation on capacity basis. The partner search and evaluation as to time, quantity, and costs, can be considered as too time-consuming by means of conventional methods due to the lack of market transparency. The search of potential partner companies in business directories, Internet and other media goes along with high costs. The dynamic environment in which companies are operating deteriorates the problem. To remain competitive, companies need a quick decision support.

Participating in such networks for external outtasking, there exists the problem of adequate data release. Ideally, capacity data of all participants in the network should be gathered so that an ideal scheduling and rescheduling can take place. This is a very unrealistic scenario. The disclosure of less information, e.g. company name and production program, provides hardly the desired potential partners. Thus, a method and IT support is necessary which works with less data but finds on that basis a significant number of partners.

Currently there is an insufficient IT support for such a case. On the one hand there are technical support options for a company-wide capacity balancing, but they are not accessible to outside companies and were not designed for such cases. On the other hand, for such open networks exist especially web-based solutions, which however act as listings and do not represent integrated solutions with minimal interfaces.

Thus, an instrument is necessary which provides standardized decision rules and procedures for short-term avoidance of such capacity problems. Here, the existence of a capacity exchange system is helpful in which required capacities of participating companies are accessible with fewer interfaces to enable rapid capacity search. For this reason, this paper deals with this promising approach of an integrated cross-company capacity exchange. 


\section{Existing Concepts}

In connection with the integrated cross-company capacity exchange existing research can be classified in the categories of "in-house solutions", "inter-company exchange solutions", "solutions for strategic networks" and "solutions for project networks and virtual enterprises". For this, there is a variety of systems and approaches. Below only relevant concepts, technical solutions, and methods are presented and analyzed.

As to in-house solutions ERP and MES systems can be stated. Against the background of the integrated capacity exchange ERP systems provide in principle the possibility to communicate with other companies in terms of order placing, but extensive interface programming for all parties is required for participating in electronic marketplaces. MES can make a contribution to that extent that they demonstrate on the basis of accurate and detailed capacity allocation the exact over or under load, which can be seen as an input for the capacity search. So these solutions enable no crosscompany capacity search and exchange. However, they are useful as data provider for the provision of the capacity search.

Apart from that there exist concepts concerning sharing of critical resources in a manufacturing system. In their paper Armetta et al. (2007) describe inspired by the stigmergic way of communication of insects an approach to elaborate complex exchanges between so-called Stigmergic Negotiating Agents for the critical resource sharing problem.

Besenfelder et al. (2013a \& 2013b) developed a decision-support system for the tactical production planning process by creating a data structure that is independent of the respective IT infrastructure of the partners and is called 'capacity corridors' to ask suppliers in the mid-run for their availability that will be taken into consideration by the own production planning tool. 
Sousa et al. (2004) present a prototype system named Fabricare for the scheduling of manufacturing orders based on holonic manufacturing systems using extended logic programming. The system is agent-based and composed of several holons that cooperate among themselves using constraint propagation in order to guarantee the precedence relationships between the different operations participating in the same task.

These works concentrate on in-company production systems and have therefore a narrow focus against the background of the topic of this paper. But the application of multi-agents is a promising approach which will be considered in the following.

Besides these approaches, there exist intercompany exchange solutions which are mainly seen as market places. Conventional electronic exchange concepts or marketplaces exist for offering and finding free capacities. In this case according to the considered object, partners, machine capacity, freight, and service markets can be distinguished. This exchange types are characterized by a rudimentary integration in the context of the present topic. Partner exchanges are company directories in which companies can present themselves with their skills and services, without considering capacity explicitly. Here companies are matched for different purposes. Furthermore, there are so-called machine capacity exchanges, which are mostly developed as closed platforms based on membership. Such platforms are in general virtual blackboards for centrally offering of machine capacities. Here, companies can present their machinery and/or their free capacities. There are also freight exchanges, which are common in the transportation and logistics industry and offer specific services for them.

In general, most of the exchange concepts described here are virtual blackboards on which hardly automated collaboration processes take place. The integration and consistency of data flow is not considered in these solutions. However, the 
agglomeration of firms in such open and closed exchange platforms represents a notable measure in terms of the network model of the integrated capacity exchange.

There also exist network-wide approaches and solutions for strategic networks, like Advanced Planning Systems (APS), which are primarily used for planning of different company locations and branches of a holding and are therefore not suitable for the present case. Furthermore, APS are not applicable in cross-industry networks.

There are also works concerning configuration and coordination of strategic partnerships in networks. Camarinha-Matos \& Afsarmanesh (2007) propose a comprehensive modeling framework towards the elaboration of a reference model for collaborative networks taking into account the exogenous perspective of life cycle. An identification of the most promising approaches among a large number of modeling tools and theories developed in other disciplines is made and mapped into four dimensions of an endogenous perspective of collaborative networked organizations: structural, componential, functional, and behavioral.

Furthermore, Chan \& Chung (2002) develop in their paper a framework to develop an enterprise information portal for contract manufacturing. This framework is only applicable to suppliers in the supply chain.

Moreover, particularly with the centralized approaches specific and unresolved issues in the present case are obvious, e.g. the complex interweaving of network enterprises with conflicting relations, rejection of exchange of trusted information, lack of acceptance of central plans, and lack of organizational structures. An extension of the planning approaches of strategic networks to lateral networks is therefore not suitable to achieve economically efficient and implementable capacity coordination between independent organizations. 
In addition to strategic networks there are also approaches and solutions which were developed for project networks and virtual enterprises. Several approaches for virtual enterprises focus strongly on their configuration, in which standardized methods are developed, so that appropriate cooperation can arise. For example, CamarinhaMatos \& Pantoja-Lima (2001) present in their paper a workflow-based approach to support coordination in virtual enterprises by developing the appropriate software modules. The adopted model considers on flexibility and configurability as the key aspects to cope with the large diversity of expectable scenarios and operating policies in the virtual enterprise environment. Further contributions for the effective and efficient configuration of virtual organizations are presented by Cheikhrouhou et al. (2013) and Camarinha-Matos et al. (2009). Apart from that, there are papers dealing with further relevant aspects of virtual organizations. Warner and Witzel (2004) and Yasir et al. (2011) worked on the coordination of virtual organizations. Baldo et al. (2009) focus on partner selection, Ahuja and Carley (1999) deal case-study based with the communication structure of a virtual organization. Wang et al. (2011) provide a semantic interoperable environment to enable automatic negotiations within virtual organizations. Chih-Yao et al. (2012) developed a simulation-based joint procurement process model for virtual organizations. Orman (2009) focuses on a design approach and develops a technology infrastructure on the basis of electronic services, formal semantics, and multi-dimensional ontologies. Sanchez-Anguix et al. (2011) examined the distribution of agents across the computational resources based on a genetic algorithm in an agent-based virtual organization.

Generally, the works dealing with virtual organizations address similar problems as of the cross-company capacity exchange. Nevertheless, since virtual organizations are actually closed networks even if it has a temporary nature, competencies are 
considered in these cases, whereby capacities are not explicitly in the focus.

Complementary competencies at the level of program planning will be combined. An analysis of the capacitive feasibility is not considered.

In summary, it can be stated that the abovementioned approaches have been analyzed against the present idea. Here, the important question was how existing approaches manage the network-wide coordination of capacity adjustments. It becomes evident that the idea of this paper is not addressed yet.

\section{Special Requirement for Cross-Company Capacity Exchange}

The analysis of existing concepts showed that none of the approaches cover the need stated above. So, the development of a novel tool enabling cross-company capacity exchange is justified. To do so, the special requirements for such a tool have to be worked out in the first instance. Since no direct customer is addressed which this tool is developed for, requirements have to be derived more general. For this, it is appropriate to look at success factors of similar tools in order to derive the special requirements from them. Especially for this issue, the success factors can be divided into structural, methodical and technical success factors which corresponding requirements can be derived from (s. Table 1).

Structural success factors relate to the shaping of the organizational structure of the system solution and the enterprise network. The size of the network as an important structural factor of success is based on the critical mass of participating companies, since the integrated capacity exchange is only considered to be effective in a large number of vertically and horizontally networked firms. The sustainability is for the sustainable use of the integrated capacity exchange also crucial. The company network must be constant over an extended period, as companies access only on demand and sporadically. Participating in such a network based on capacity exchange, the 
individuality in terms of entrepreneurial independence has to be guaranteed avoiding central orders or restrictions. The neutrality of the integrated capacity exchange refers primarily to the operator of the system, which is acting trustworthy, and as an independent mediator in case of technical and methodological problems.

\begin{tabular}{|c|c|c|}
\hline Dimension & Success Factors & Special Requirements \\
\hline \multirow[t]{4}{*}{ Structural } & Network size & Simple scalability \\
\hline & Sustainability & Self-growing database \\
\hline & Individuality & Clear competence profile \\
\hline & Neutrality & No central controller \\
\hline \multirow[t]{3}{*}{ Methodical } & Planning assistance & Simple recognition of capacity requirements \\
\hline & Economic advantageousness & Fast and cheap search process \\
\hline & Decision support & Providing of all relevant data \\
\hline \multirow[t]{7}{*}{ Technical } & Data security & local data management \\
\hline & User friendliness & Easy installation and usage \\
\hline & Communication security & Encrypted data transfer \\
\hline & Robustness & Alternative search options \\
\hline & Consistency & Timeliness of data \\
\hline & Standardized interfaces & $\begin{array}{l}\text { Application of a standard agent } \\
\text { communication environment }\end{array}$ \\
\hline & Platform independence & $\begin{array}{l}\text { Application of platform independent } \\
\text { programming language (Java) }\end{array}$ \\
\hline
\end{tabular}

Table 1: Success factors and derived requirements 
The methodical success factors relate to the scope of the methodology for the configuration of cooperation. Firstly, the system has to give assistance in the planning and scheduling of capacity requirements. As a further decisive methodical success factor, the economic advantageousness can be seen. Companies will use the integrated capacity exchange only if the benefits outweigh the costs. In particular time and economic indicators are of relevance. Moreover, the decision support for the choosing the right partner is also an important methodical success factor.

The technical success factors ultimately relate to the design of the technical solution of the integrated capacity exchange system. Here, the data and communication security, user-friendliness of the technical solution, the robustness of the system with stable working modules, the consistency of the data with a seamless media data flow, standardized interfaces, and platform independence are of vital importance.

Based on these success factors, corresponding special requirement can be assigned. Table 1 shows the requirements which have to be taken into consideration during the development of the integrated capacity exchange system.

\section{General Concept of the Integrated Capacity Exchange System}

The integrated capacity exchange system combines independent, geographically distributed participants and dispensing with central control. For this, a technology is needed that is suitable for the realization of the distributed structure of the integrated capacity exchange. In recent years the multi-agent technology emerged, as seen above, in which actually existing entities are represented as software agents for complex situations, which together handle certain tasks autonomously.

So, the basic structure of the integrated capacity exchange system takes this technology into consideration and includes a database, a central web interface, a 
communication client and a planning system that communicate with each other by means of agents. The modular design of the integrated capacity exchange system ensures that companies with different information technology equipment can participate in the exchange of services. Within the integrated capacity exchange system, the individual modules interact with each other.

The software client is used for inter-company communication in the case of capacity search and is the link between the planning system, database, and clients of other companies. The appropriate outtasking operation will be selected and specified, the search for potential partners started and then listed, and finally the order submitted. Capacity providers submit their offers and price bids by means of the client.

In detail, the procedure can be depicted as follows. A company, that cannot schedule a job or an operation, can start the outtasking by means of the software client. If using the integrated planning tool in case of failure of scheduling of the corresponding order, outtasking will be proposed without additional prompts by means of a dialog box. If the capacity demander is willing to outtask a job, this specific one is selected from the list of orders. Subsequently, the corresponding capacity requirements are specified by means of the software client. Thereafter, the capacity request is sent automatically. For this purpose, in the first step the database in which all participating companies are registered is scanned for potential companies. Potential companies are those that could handle the outtasking operation due to the possession of certain machine tools and the presence of manufacturing expertise. At the same time the outtasking order is listed in the outtasking storage list of the capacity demander.

After receiving information on potential companies from the database, the companies are contacted by the software client to get their temporal availability, which in turn respond to the capacity-seeking company through their software clients. If a 
company receives a capacity request, the agent of the contractor visualizes the order in the order box, which is then to be evaluated. In this display window for incoming orders there are essential information as to the capacity demanding company as well as the possibility to simulate scheduling in order to check availability. Here, the times are blocked or reserved on the affected machine.

Companies that signal a temporal availability are listed with all the other feedbacks from other providers in the potential partner list. They get in the next step to the order specification documents, such as technical drawings, in which they can now check their technical competence, give a price level, and submit a response by means of their software clients. After submitting an offer this will now be re-listed giving the price in the window of the potential partner list along with the offers of other companies. The capacity-seeking company reviews in turn all submitted offers and selects in the next step a company, to whom he would like to award the contract. In this way, an agent is started, which announces the company the relevant procurement. Finally, this job will be scheduled by both partners. The detailed sequencing of this process is visible in Figure 1. 


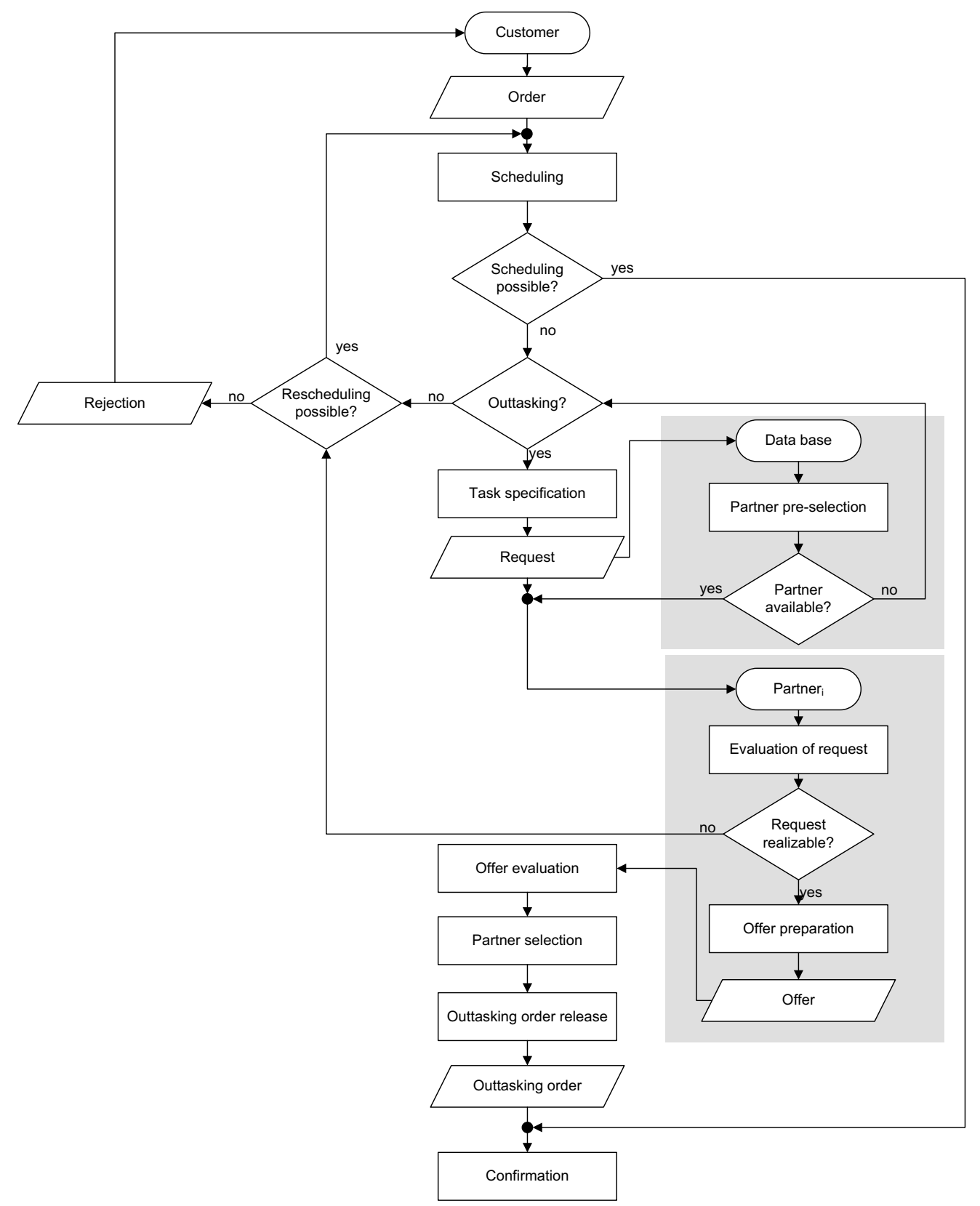

Figure 1: The specific procedure model of the cross-company capacity exchange

\section{$5 \quad$ Specification of the Outtasking}

A triggered order processing due to constraints and interferences in the cross-company capacity exchange using this system can be detailed with reference to the features and their values. For detailing and specifying the order processing the feature categories initiation, capacity, scheduling, product, and process can be distinguished, which are in a logical connection to each other. 


\begin{tabular}{|c|c|c|c|c|c|}
\hline Category & Characteristic & \multicolumn{4}{|c|}{ Characteristic Attributes } \\
\hline Initiation & Demand release & $\begin{array}{c}\text { reactive } \\
\text { unplanned }\end{array}$ & \multicolumn{2}{|c|}{$\begin{array}{l}\text { reactive } \\
\text { planned }\end{array}$} & proactive \\
\hline Capacity & Type of capacity requirement & congruent & \multicolumn{2}{|c|}{ complement } & substituting \\
\hline \multirow{2}{*}{ Scheduling } & Planning horizon & Short term & \multicolumn{2}{|c|}{ term } & Long term \\
\hline & Planning stages & Single stage & \multicolumn{2}{|c|}{$\begin{array}{l}\text { multistage } \\
\text { sequential }\end{array}$} & $\begin{array}{c}\text { Multistage } \\
\text { parallel }\end{array}$ \\
\hline \multirow{2}{*}{ Product } & Complexity & \multicolumn{2}{|c|}{ Standard parts } & \multicolumn{2}{|c|}{ Complex parts } \\
\hline & Provision of material & \multicolumn{2}{|c|}{ With provision } & \multicolumn{2}{|c|}{ No provision } \\
\hline \multirow[b]{2}{*}{ Process } & Process complexity & \multicolumn{2}{|c|}{ Standard process } & \multicolumn{2}{|c|}{ Special process } \\
\hline & Number of Processes & \multicolumn{3}{|c|}{ One process } & $\begin{array}{c}\text { Several } \\
\text { processes }\end{array}$ \\
\hline
\end{tabular}

Table 2: Morphology of the order processing within the cross-company capacity exchange (Uygun 2012)

The morphology in Table 2 allows the determination and specification of three ideal-typical categories of contracts that may occur in the cross-company capacity exchange. According to Uygun \& Ilie (2018) these are the

- $\quad$ capacity-related outtasking (CRO),

- capacity-related procurement (CRP) and

- technology-related outtasking (TRO). 
The capacity-related outtasking is relevant in case of capacity problems (break down, failure, etc.). The affected process will normally continue to be conducted inhouse. Thus, a temporary by-pass is installed. The need is here triggered reactively unplanned due to the sudden occurrence of a problem. Thus, congruent or similar capacity is required. Due to the sudden occurrence of the problem, a short-term singlestage adaptation planning is required. Primarily, standard parts are outsourced, whereas semi-finished parts are supplied as input. This process also has a low complexity, which allows its relatively fast implementation.

As part of the capacity-related procurement a capacity adjustment is performed. On the basis of existing orders the capacity requirement is determined, which necessitates a capacity-related procurement, if the internal capacity level is exceeded. The planning of the capacity-related procurement is thus triggered reactively. Here, depending on the problem either complementary/supplementary or congruent/similar capacities are considered. The planning horizon is days to weeks or a few months, with single to multi-stage planning. The products may be standard or complex, which materials can be supplied or not. Thus, the outtasked processes can be standard or special in terms of technology. One or more processes are outsourced.

If processes or operations of a particular technology are outsourced, a technology-related outtasking is considered. Here, economic considerations play a role. The in-company processing of these processes is unfavorable in terms of cost, making these processes eligible to outtasking to specialized external production service providers, e.g. surface coating. The corresponding capacities are not maintained internally (anymore). The demand is determined in advance. Depending on the company's situation with the presence of own capacities, capacity requirements can be complementary or substituting. In the former case, there are no own capacities, whereas 
in the latter case own capacity are given up. Since this kind of subcontracting must be well thought out, the planning horizon takes several months. The planning can include one to several steps.

These types of contracts within the cross-company capacity exchange will be addressed below in the application to find out which type is used more frequently.

\section{Communication Structure}

The development of the communication structure of the multi-agent system, which uses the above-described components of the system is performed on the basis of the PASSIprocedure model, according to Cossentino et al. (2005), ICAR/CNR (2010) and Chella et al. (2006). For this, the phases of the functional system description, agent identification, role identification, task identification, ontology description, role description, multi-agent implementation and implementation of individual agents are run and completed with the application configuration.

In the first phase, the functional description of the total system is performed based on the so-called use case diagrams. In preparing these and other diagrams the UML standard will be referred to.

Agents who are behind the identified functions can be grouped, which is necessary for the phase of the agent identification. Here, the functionality of each agent will be defined. An agent includes one or more individual functions. Agents can exchange messages with each other, send important information to place an order, add a machine to the database, match the company's login information with the database and look for potential companies that have a required machine.

The agents ClientAgentLogIn, ClientAgentMachineAdd, ClientAgentJob and ClientAgentMachineDelete are relevant for both the capacity provider and demander. The ClientAgentJob collects all offers of capacity providing companies. On the part of 
the client the ManagerAgentCustomer is a central agent that is started automatically or manually. After this, it then enters into contact with the database first and then with companies that have the desired machine tool or competence. Henceforth, it is the central contact partner for communication with the appropriate agent of potential partner companies via the mediating agents ClientAgentJob and DBAgentJob. It sends directly the job data to potential capacity providers and receives offers from these through intermediary agents that it then visualizes for the capacity demander.

On the part of the capacity provider the ManagerAgentProvider is the counterpart of the ManagerAgentCustomer. The ManagerAgentProvider maintains contact with the appropriate agent of the capacity demander and the intermediary agents, by visualizing the requests, submitting offers, transferring the job to schedule, and sending the final confirmation. In the database, the DBAgentMachineListener which selects potential companies with the right machine from the database and the DBAgentJob exist. The latter is a mediator and holds a bridge function between the manager agents of the customer and provider. It collects the offers and the price bids of each manager agent of potential partner companies and forwards it to the manager agent of the customer. For completeness, there are the database agents DBAgentLogin, DBAgentMaschineAdd and DBAgentMachineDelete which interact with the aforementioned manager agents.

The next phase, namely the role identification, is based on the identification of all the possible scenarios of the actions of the identified agents to fulfill their function in the context of the multi-agent system. These are illustrated by the well-known UML sequence diagrams in which the roles of each agent can be seen. The exchanged messages in the sequence diagrams can come from external (e.g. manual entry) or from the internal communication between the agents. A message in this case defines the 
executed role of an agent. The data of such a message are specified in the following ontology description phase. In addition to this, also non-agents, such as the integrated planning tool, are listed.

After the phase of the roll identification the phase of the task specification follows, in which the individual behavior, and tasks of the agents are presented based on activity diagrams. A task includes certain functionalities that form a logical unit of work. A transition between the various activities can mean the communication between agents, but also the transition to a new state.

The semantics of the multi-agent system is described in terms of explicit ontologies for which class diagrams are used. On the one hand, the domain ontology, in which the existing entities are represented by classes, and on the other hand communication ontology with the agent knowledge and the communication relationship of the agents are considered.

The domain ontology is described as an XML scheme. It represents a class diagram describing the different classes of the multi-agent system. The domain is represented using concepts, actions, predicates, and relationships between them. They various classes may consist of simple data types, such as integer or string. With the help of the class diagrams, the ontologies of the agents are defined, described and shown. First, the ontology of the domain is described, showing the components involved in the software by individual classes, such as order, machine, company, customer, provider, offer, and database. The elements in this diagram define the agent knowledge and the ontology of communication.

The communication ontology is used for the description of the agent knowledge and communication, where each agent is represented by a single class. This includes his 
knowledge. The agent communication is specified regarding ontology elements, language, and protocols.

In the role description the life cycle of an agent with its roles, his need for cooperation and its communication is modeled. The individual roles of the agents in the society and the tasks for each role are detailed. Here, individual roles are represented by classes. Accordingly, the class diagram for such presentation is applicable. This class diagram is extended by the attributes of the classes in the next step, which results in the multi-agent structure.

Within the multi-agent implementation the structure and the behavior of the agent are defined, which influence each other. It represents a model of the system architecture based on classes with the appropriate attributes and methods and includes the structure definition and the definition of behavior.

Within the single agent implementation, the structure and behavior of each agent are defined, whereas the structural and behavioral definitions are also distinguished here. For each agent, a class is defined by which the inner structure of the agent is determined with its main and sub-classes necessary for the task identification.

In the last phase of the use configuration, the positions of the agents in the distributed system are defined. There is a distinction between database and users, the latter are further subdivided in customer and provider.

In the database all corresponding database agents are accommodated which interact with the agents of the users. User data, machine data as well as order data are exchanged.

Eventually, it has to be stated that each company has both the ManagerAgentCustomer and the ManagerAgentProvider, since a company can function both as capacity demander and capacity provider. 


\section{$7 \quad$ Application and Results}

To validate the developed integrated capacity exchange system a network of companies has been built up. For validation, 22 companies initially have agreed. Over a period of six month the prototype of the developed integrated capacity exchange was used within this network. The number of participants grew over time up to 37 after six month.

Figure 2 shows the results of the order processing types described previously. Placing an outtasking request, one of these three types had to be checked in order to proceed. So, it becomes obvious that the capacity-related outtasking (CRO) was the most popular type of outtasking within this network whereas the capacity-related procurement (CRP) ranks as least. Technology-related outtaskings were considered especially for painting and surface treatment.

Looking deeper into this network it becomes evident that there exist predomionantly subcontractors. Interviewing several of them, their existence could be a possible explanation for the high number of CRO, since many of the interviewed subcontractors acquire orders at any cost without scheduling in detail and relying on their flexibility to scale shifts. In cases of overload they seek partners to handle the order load in time.

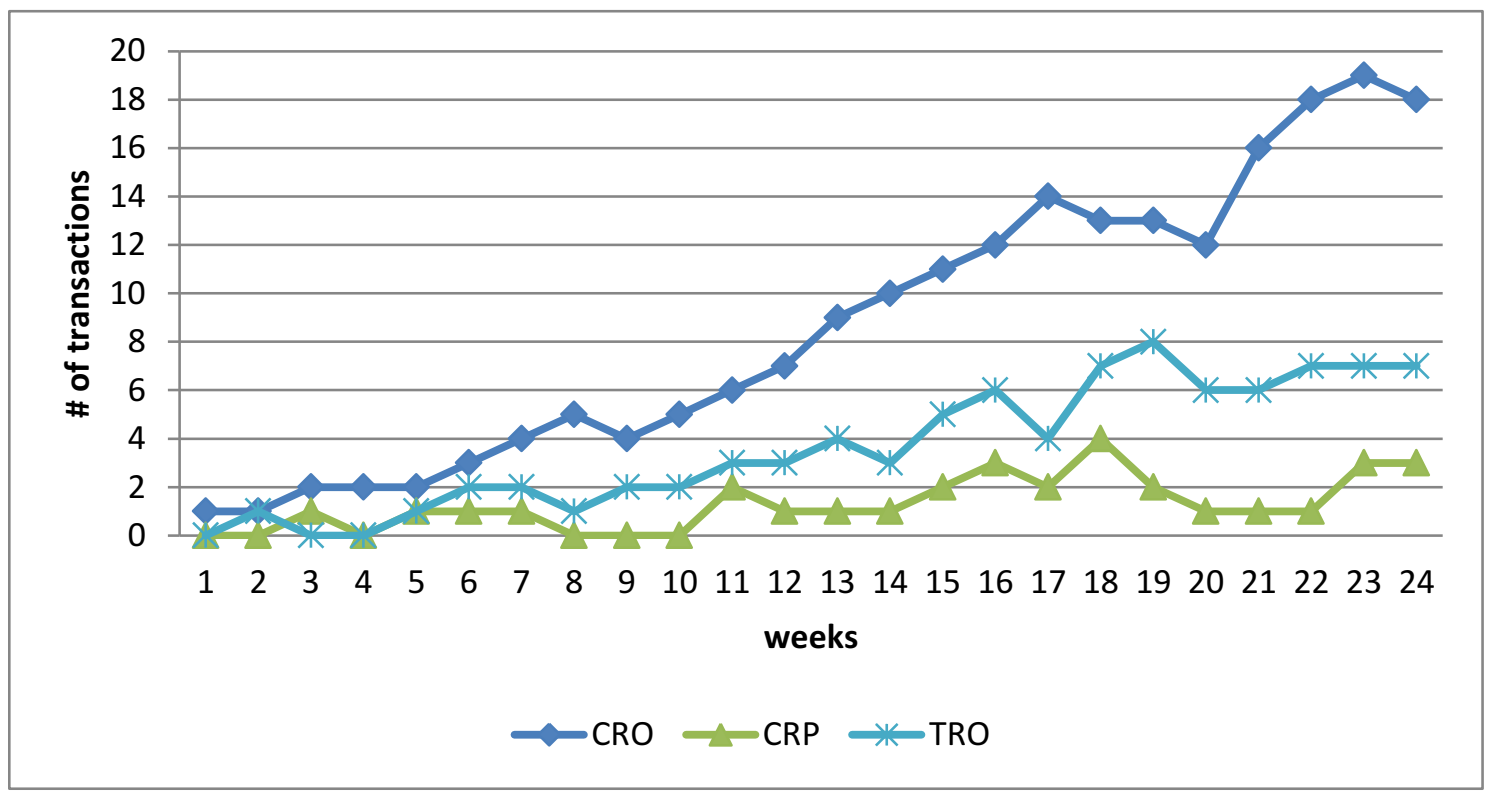


Figure 2: Number of order processing types

Based on the mentioned interviews further particularities were observed.

Although the integrated capacity exchange enables fully automatic search for capacity, manual steps, which are also provided, were used in particular for the assessment of the potential partner and final confirmation of the outtasking. The manual phases can have different lengths. This depends mainly on the familiarity, number of transactions and also the quality of the outtasked work processes. The more intense these factors are, the lower the effort for manual transaction phases. In terms of familiarity, a comprehensive analysis and evaluation of the partner can be dispensed with, when partners are available, with which companies have already worked successfully and satisfactorily in the past. An extensive partner analysis can also be waived for work processes with low value. With frequent transactions, a cost reduction can occur due to the learning effect.

Basically, trust was named as an important aspect for inter-company cooperation in general and for the cross-company capacity exchange in particular. To build cooperation, a certain level of trust is required. It can be characterized as a stabilizing mechanism. The existence of trust, however, facilitates the coordination and conflict handling of the operating companies in the network which also supports an open exchange of information between partners. Trust may even make contracts unnecessary to a certain degree.

\section{Conclusion}

In general, cross-company capacity exchange which is enabled by the developed tool is actually an electronic market acting according to established rules, which provides buyers and sellers free quantitative capacities. Depending on the lead time, this can be a 
spot market or a futures exchange. The capacity exchange can be considered as a free exchange which enables regular and non-localized trading.

Different from the concepts of Internet marketplaces and auction platforms which merely bring together buyers and sellers, the developed cross-company capacity exchange system embraces the planning, searching, and negotiation. Based on the parameters trade objects, resources, process, planning, and data, the developed system can be distinguished from the other analyzed systems. Regarding the performance object primarily production-related services are addressed. The considered resources are machine tools. Based on the feature category process, the cross-company capacity exchange system is primarily used in the context of the business process of order management. In certain cases a deployment could also be relevant in the product development phase. Within the framework of planning, the cross-company capacity exchange system includes primarily tactical and operational planning functions. A special feature of the cross-company capacity exchange system is the decentralization of planning, so that capacity planning is still performed in-company, and only necessary information are shared. Regarding data, the cross-company capacity exchange runs with a low volume of data. Only required information will be sent. Therefore no critical content is considered. Here, unstructured or highly structured database entries are possible. The former allows specific and individual details that make it difficult to search for entries, whereas the latter can prevent this. The cross-company capacity exchange system strongly considers in this context the structured data.

The cross-company capacity exchange system generates a network of participating companies which act on two levels; the lower level of the enterprise network and the upper level of cooperation. In the level of the enterprise network all coopted companies are combined, which can also be named as passive element. This 
network cannot be controlled or planned in terms of classical organizational control. However, it can be cultivated by setting the appropriate environment, such as through access requirements, so that the network development can be influenced. The level of cooperation includes job-related networking and has thus clearly defined content and limits and is thus principally controllable. On the level of cooperation arises either a bilateral cooperation or a job-related network and can be described as an active element.

Finally, it can be stated that the integrated capacity exchange meets very well all the structural, methodological, and technical requirements described initially.

Future research may be done on performance measurements to evaluate the transactions and use those as decision criteria for future exchanges. For this, the framework developed in Uygun \& Schmidt (2011) will be adjusted.

\section{References}

Ahuja, M.K., and K.M. Carley. 1999. "Network Structure in Virtual Organizations". Organization Science 10 (6): 741-757.

Armetta, F., S. Hassas, S. Pimont, and O. Lefevre. 2007. "Towards the Control of Emergence by the Coordination of Decentralized Agent Activity for the Resource Sharing Problem". In Engineering Self-Organising Systems: Lecture Notes in Computer Science, edited by S.A. Brueckner, S. Hassas, M. Jelasity, and D. Yamins, 132-150. Berlin: Springer.

Baldo, F., R. Rabelo, and R. Vallejos. 2009. "A framework for selecting performance indicators for virtual organisation partners' search and selection”. International Journal of Production Research 47 (17): 4737-4755. 
Besenfelder, C., Kaczmarek, S., and Y. Uygun. 2013a. "Process-based cooperation support for complementary outtasking in production networks of SME". International Journal of Integrated Supply Management 8 (1/2/3): 121-137.

Besenfelder, C., Uygun, Y., and S. Kaczmarek. 2013b. "Service-oriented integration of intercompany coordination into the tactical production planning process". In Robust Manufacturing Control, edited by K. Windt, 301-314. Berlin: Springer.

Camarinha-Matos, L.M., and C. Pantoja-Lima. 2001. "Cooperation coordination in virtual enterprises". Journal of Intelligent Manufacturing 12 (2): 133-150.

Camarinha-Matos L.M., and H. Afsarmanesh. 2007. "A comprehensive modeling framework for collaborative networked organizations". Journal of Intelligent Manufacturing 18 (5): 529-542.

Camarinha-Matos, L.M., A.I. Oliveira, M. Sesana, N. Galeano, D. Demsar, F. Baldo, and T. Jarimo. 2009. "A framework for computer-assisted creation of dynamic virtual organisations". International Journal of Production Research 47 (17): 4661-4690.

Chan, M.F.S., and W.W.C. Chung. 2002. "A framework to develop an enterprise information portal for contract manufacturing”. International Journal of Production Economics 75 (1-2); 113-126.

Cheikhrouhou, N., A.H. Tawil, and A. Choudhary. 2013. "Modelling competence-based virtual organisations using the unified enterprise competence modelling language". International Journal of Production Research 51 (7): 2138-2159.

Chella, A., M. Cossentino, L. Sabatucci, and V. Seidita. 2006. "Agile PASSI: An Agile Process for Designing Agents”. International Journal of Computer Systems Science \& Engineering" 21 (2). 
Chih-Yao L, C. Yu-Teng, and C. Kuo-Ting. 2009. „A Multiple-Agent Joint Procurement Process Model for Virtual Organizations: A Simulation Study". International Journal of Management 29 (1): 352-367.

Cossentino, M, S. Gaglio, L. Sabatucci, and V. Seidita. 2005. “The PASSI and Agile PASSI MAS Meta-models Compared with a Unifying Proposal”. In Multi-agent systems and applications IV - 4th International Central and Eastern European Conference on Multi-Agent Systems - Proceedings edited by M. Pechoucek, P. Petta, and L. Varga. 183-192. Berlin: Springer.

ICAR/CNR. 2010. "PASSI". Accessed December 12.

http://www.pa.icar.cnr.it/passi/index.html.

Orman, L.V. 2010. "Virtual Organizations as Electronic Services". Communications of the Association for Information Systems 201024 (Article 40): 701-718.

Sanchez-Anguix, V, S. Valero, and A. Garcia-Fornes. 2011. "A Genetic Approach for Long Term Virtual Organization Distribution”. International Journal on Artificial Intelligence Tools 20 (2): 271-295.

Sousa, P., C. Ramos, and J. Neves. 2004. “The Fabricare system: a multi-agent-based scheduling prototype". Production Planning \& Control 15 (2): 156-165.

Uygun, Y. and A. Schmidt. 2011. "Performance Measurement for Interorganisational Collaborations of SMEs". In Dynamics in Logistics, edited by H. J. Kreowski, B. Scholz-Reiter, K. D. Thoben. 169-189. Berlin: Springer.

Uygun, Y. 2012. “Integrierte Kapazitätsbörse - Entwicklung eines Instrumentariums für den Handel mit Maschinenkapazitäten in regional-lateralen Unternehmensnetzen". Dortmund: Verlag Praxiswissen. 
Uygun, Y. and M. Ilie. 2018. “Autonomous Manufacturing-related Procurement in the Era of Industry 4.0". In Digitalisierung im Einkauf, edited by F. Schupp and H. Wöhner. 81-97. Wiesbaden: Springer.

Wang, X.H., T.N. Wong, and G. Wang. 2011. "Knowledge representation for multiagent negotiations in virtual enterprises". International Journal of Production Research 49 (14): 4275-4297.

Warner, M., and M. Witzel. 2004. Managing in virtual organizations. London: Thomson Learning.

Yasir, M., M.T. Abdullah, and A. Majid. 2011. "Coordination in Virtual Organization: A Strategic Solution for Sustainable Development". Asian Journal of Business Management 3 (1): 18-22. 